\title{
COMMISSIONED REVIEW
}

\section{Insights into root development from Arabidopsis root mutants}

\author{
P. N. BENFEY ${ }^{1} \&$ J. W. SCHIEFELBEIN ${ }^{2}$ \\ ${ }^{I}$ Department of Biology, New York University, 1009 Main Building, New York, NY 10003, and ${ }^{2}$ Department of Biology, \\ University of Michigan, Ann Arbor, Michigan 48109-1048, USA
}

\begin{abstract}
The root provides a useful system for the analysis of plant organ formation. Mutations that affect root development and physiology have been identified in Arabidopsis thaliana. Affected processes include embryonic root formation, cell expansion, cell differentiation and response to environmental stimuli. Analysis of these mutations is providing insight into fundamental questions of plant development.
\end{abstract}

Keywords: Arabidopsis thaliana; organogenesis; meristem; stimulus-response.

\section{INTRODUCTION}

Until recently, root development and physiology were studied largely without the aid of genetics. There was a simple reason for the paucity of genetic data: the identification of mutant structures generally required unearthing the roots. The use of Arabidopsis thaliana as a model genetic organism has greatly facilitated the genetic analysis of root development. The Arabidopsis seedling is small enough to permit growth of large numbers on nutrient media plates. When the plates are oriented vertically, the roots grow along the surface of the agar media and abnormalities can be observed easily. This has facilitated genetic screens for mutations that affect the development and physiology of the root.

We will provide an introduction to root development in Arabidopsis, then review the mutations that disrupt normal development or physiology.

\section{ROOT DEVELOPMENT IN ARABIDOPSIS}

The Arabidopsis zygote divides to form two cells of different sizes. Most of the embryo proper will be derived from the smaller apical cell. The larger basal cell divides to form the suspensor and the hypophysis (Mayer, Buttner \& Jurgens 1993). The hypophysis goes through a series of stereotyped divisions to form a part of the embryonic root meristem and root cap. All the other cells of the embryonic root are derived from the apical cell (Dolan et al. 1993).

Correspondence: Dr Philip N. Benfey, Department of Biology, New York University, 1009 Main Building, New York, NY 10003, USA.
Anatomical analysis of mature embryos revealed a relatively simple organization of the embryonic root (Dolan et al. 1993). Putative initial cells were identified by following cell files towards the tip of the root. It appears that there are four distinct classes of initials. One set of 12 initial cells gives rise to the columella or central portion of the root cap (Fig. 1b). Flanking these are another 16 cells that are the initials for both the lateral root-cap and the epidermis. Internal to and above the epidermal/root cap initials are eight cells that give rise to both the cortex and endodermal cell layers. At the base of the stele, there are eight initials for the pericycle and vascular cylinder (Fig. 1).

There are four cells at the centre of the root meristem. DNA labelling experiments revealed that these cells rarely divide while the cells surrounding them have division rates consistent with their roles as initials. Therefore, the four central cells constitute the quiescent centre of the Arabidopsis root (Fig. 1). The quiescent centre was first identified in maize roots as a population of cells located at the centre of the meristem that has a very low rate of cell division (Clowes, 1958; reviewed in Feldman, 1984). It has been proposed that the cells of the quiescent centre serve as replacements for initial cells that occasionally enter the differentiation pathway (Barlow, 1976).

Analysis of the seedling root has disclosed a similar simplicity of organization. From transverse sections, it was found that there is a single layer of the epidermis, cortex, endodermis and pericycle (Fig. 2). These layers have radial symmetry, and the endodermis and cortex have an invariant number of eight cells each in the primary root (Dolan et al. 1993; Benfey et al. 1993). In the epidermal layer, there are two cell types: those that make root hairs and those that are hairless. The root hair cells are always found above the junction of two internal cortex cells.

Root growth is fairly uniform, and consists of continuously producing the same cell types in approximately the same numbers and in the same places. This results in the different stages of development being present in zones along the root axis at all times of root growth. Longitudinal sections through the root tip can be divided into: (1) the meristematic zone which comprises the quiescent centre and the dividing cells around it; (2) the elongation zone in which cells primarily expand; and (3) the differentiation zone in which cells attain their differentiated characteristics. Of course, there is a degree of overlap in the processes that take place in each zone. 
(a)

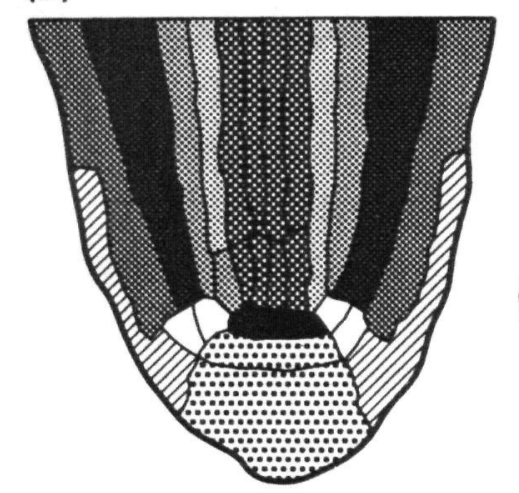

(b)

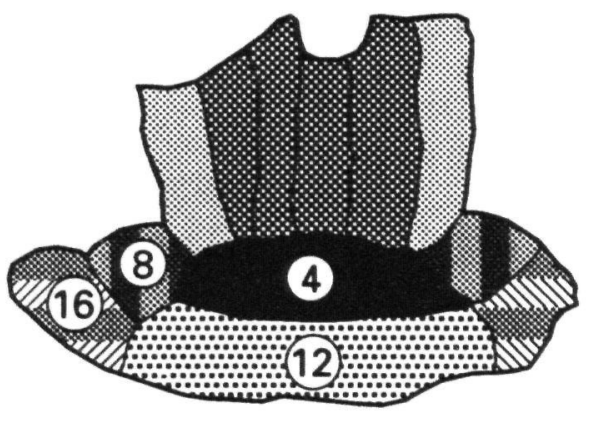

(c)

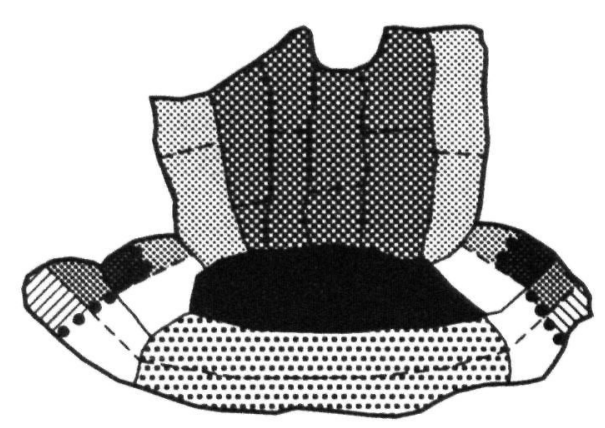

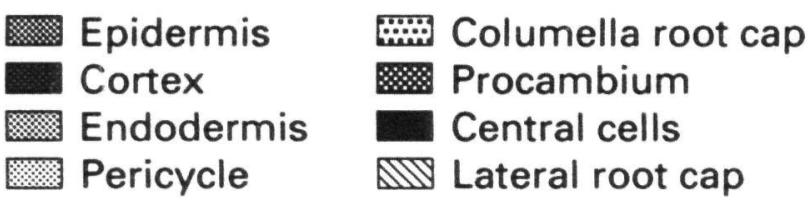

Figure 1. Organization of the embryonic root meristem of Arabidopsis: (a) schematic representation of the initials and cell files in the embryonic root; (b) magnified view of the region of the initials with the total numbers of each type of initial indicated; and (c) the proposed division patterns of the initials to generate the cell files. The order of division is indicated as: first division, dashed lines; second division, large dots. [After L. Dolan et al. (1993) Development 119, 71-84; copyright 1993, Company of Biologists.]

(a)

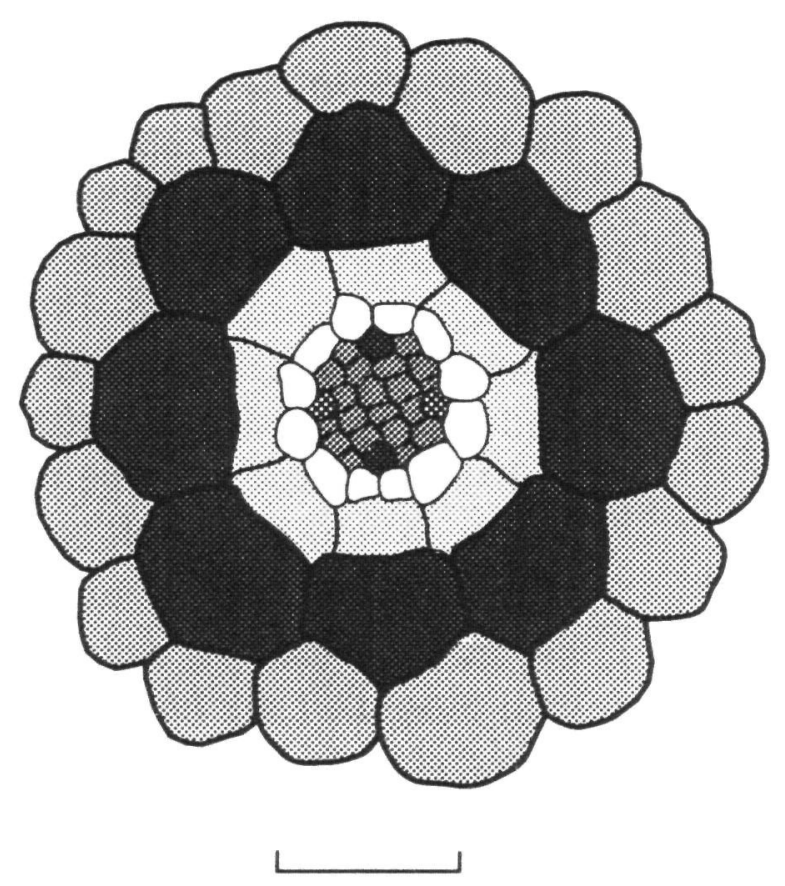

(b)

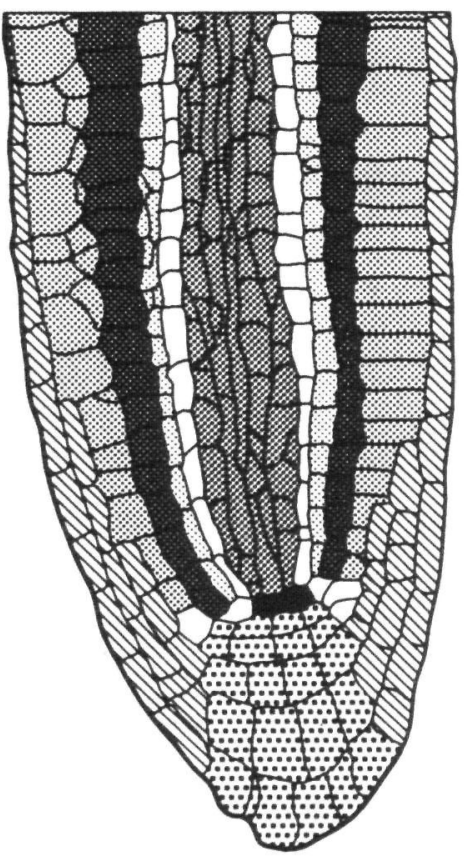

\section{Epidermis Cortex Endodermis Pericycle Protoxylem}

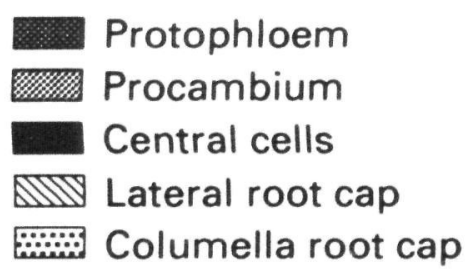

Figure 2. The seedling root of Arabidopsis: (a) organization of tissues in transverse section from differentiation zone; (b) organization of tissues in root tip. Initials for endodermis and cortex, and for epidermis and lateral root cap are in white (bar $25 \mu \mathrm{m}$ ). [After L. Dolan et al. (1993) Development 119, 71-84; copyright 1993, Company of Biologists.] 
A clonal analysis needs to be performed to confirm that the anatomical data are consistent with dynamic relationships. In the water fern, Azolla pinata, the clonal history of every cell in the root has been determined (Gunning 1982). The situation in Azolla is somewhat simplified by the presence of a single cell that serves as the initial for all the other root cells. Clonal analysis in Arabidopsis is performed using a transposable AC element that interrupts a marker gene (L. Dolan, K. Roberts and B. Scheres, personal communication). Upon excision of the transposable element, expression of the marker gene can be detected in sectors that reveal the clonal relationship among the cells.

\section{MUTATIONS THAT AFFECT EMBRYONIC ROOT FORMATION}

Two mutations that affect development of the embryonic root were identified in a large-scale screen for plants with abnormal embryonic pattern formation. The screen was based on the assumption that defects in pattern formation would not prevent germination (Mayer et al. 1991; Jurgens et al. 1991). Therefore, seedlings from mutagenized parental plants were screened for abnormal body patterns.

The gnom mutation was initially described as resulting in a seedling without shoot or root apical meristems (Mayer et al. 1991). A detailed analysis of 24 alleles indicated that the phenotype varied from a mass of cells in the form of a ball, to a seedling with a relatively normal hypocotyl but no root or shoot apical meristem (Mayer et al. 1993). Surprisingly, each allele exhibited the same range of phenotypes. The origin of the phenotypic variability was traced back to the initial defect in the embryo. In mutant embryos, the first zygotic division produces an apical and basal cell of approximately the same size as opposed to the normal asymmetric division. Subsequent divisions are highly variable, whereas in the wild-type embryo, they follow a very stereotyped pattern. It was hypothesized that the variability of the placement of the cell wall in these later embryonic cell divisions is the origin of the range of possible phenotypes (Mayer et al. 1993). This indicates that the correct placement of the cell wall in the first asymmetric division is essential for the proper formation of the embryonic root as well as other embryonic structures.

The phenotype of the monopteros mutation is somewhat more specific for the embryonic root. In weak alleles, there is no root development while the upper part of the plant appears relatively normal (Berleth \& Jurgens 1993). In stronger alleles, the hypocotyl is also missing. The defect was first identified at the octant stage of embryogenesis. At this stage, the lower tier of cells begins to differentiate from the upper tier. In monopteros embryos, this differentiation fails to take place so that the lower tier resembles the upper tier cells. In addition, at this stage, the hypophysis goes through a series of transverse and longitudinal divisions to form the quiescent centre and columella root cap of the embryonic root. In the mutant, the longitudinal divisions do not take place (Berleth \& Jurgens 1993). Therefore, it appears that the MONOPTEROS gene may play a role in coordinating the interactions between cells derived from the hypophysis and those of the lower tier to form the embryonic root.

Double mutant analysis, which indicated that gnom was epistatic to monopteros, was consistent with the observation that gnom acts earlier than monopteros in embryogenesis (Mayer et al. 1993). A mutation that has a less drastic effect on embryonic root development is hobbit. In hobbit mutants, there appears to be no root meristem, even though the surrounding embryonic root appears to be relatively normal (Scheres et al. 1993).

\section{MUTATIONS THAT AFFECT THE STRUCTURE OF THE MATURE ROOT}

The three-dimensional structure of the root is dependent primarily on the interplay of three processes. In the meristem, the timing of cell division and the orientation of the plane of cell division play important roles in defining the size and number of cells that are formed. In the elongation zone, the direction and extent of expansion determine the final shape of each cell. Mutations that affect these processes should result in roots that have abnormal lengths and/or diameters.

A mutant that makes abnormally short roots has been identified (Benfey et al. 1993). Roots of short-root appear to be initiated normally but root growth arrests prematurely. The tips of these roots appear to have no meristematic or elongating cells. It appears that all cells have fully differentiated. This mutant has a second defect that co-segregates with the determinate root phenotype. There is no endodermal cell layer in the root or hypocotyl (Benfey et al. 1993). Analysis of mutant embryos revealed that the endodermis was missing at the torpedo stage of embryogenesis indicating that this is a radial pattern defect (B. Scheres, L. Di Laurenzio and P. Benfey, manuscript in preparation). It has not yet been determined whether a causal relationship exists between the two phenotypes.

Several mutations that result in abnormal root expansion have been characterized. The cobra, lion's tail and pom pom mutants have roots that have regions that are expanded to several times the normal diameter (Benfey et al. 1993; Hauser \& Benfey 1993). A common feature of these mutations is that the degree of expansion varies along the length of the root. Another common feature is that the expansion phenotype is conditional upon the rate of root growth. When root growth is maximal, there is the greatest degree of expansion. If the growth rate is reduced (e.g. by reducing sucrose in the media or lowering the temperature), the root appears similar to wild-type (Benfey et al. 1993; Hauser \& Benfey 1993). Sections of the expanded roots revealed striking differences among the three mutants. In cobra, expansion is primarily in the epidermal layer; in lion's tail, expansion is greatest in the stele cells; and in pom pom, both the epidermis and cortex are abnor- 
mally expanded. This suggests that these mutations either affect genes that are expressed in a tissue specific fashion, or affect processes or components that are differentially distributed among the root tissues.

The sabre mutation also causes abnormal root expansion, but there is relatively little variation in the degree of expansion along the root length (Benfey et al. 1993). In addition, the phenotype is not conditional upon the rate of root growth. However, expansion again appears to be primarily in one cell layer: the cortex. The SABRE gene has been isolated and a functional analysis is in progress ( $\mathrm{R}$. Aeschbacher and P. Benfey, manuscript in preparation).

A screen for temperature-sensitive root expansion mutants led to the isolation of three lines named $r s w 1-3$ (for root swelling) (Baskin et al. 1992). The roots appear normal at the permissive temperature of $18^{\circ} \mathrm{C}$ but exhibit abnormal swelling at the restrictive temperature of $31^{\circ} \mathrm{C}$. It appears that the epidermal cell layer of $r s w 3$ is abnormally expanded. Allelism tests with the expansion mutants listed above are in progress.

Cell expansion is a complex process that must involve coordination of the formation of the internal cytoskeleton and external cell wall. It is thought that the orientation of the macromolecules located in these two structures plays a critical role in determining the orientation of expansion. In particular, the orientation of the cortical microtubules appears to influence the orientation of the cellulose microfibrils (Giddings \& Staehelin 1988). It is hoped that the genetic analysis of root expansion will provide insight into how these processes are regulated at the molecular level.

\section{MUTATIONS THAT AFFECT ROOT CELL DIFFERENTIATION}

After cells are formed by divisions at the root meristem, they must differentiate in an appropriate fashion to generate the functional root. Cell differentiation includes the many processes that define the morphological and biochemical characteristics of the mature cells. Genetic studies of the Arabidopsis root have led to the identification of many mutants with defects in cell differentiation. These mutations primarily affect the root epidermis.

Two types of cells are present in the root epidermis: root-hair cells (cells that possess a long tubular-shaped outgrowth) and hairless cells. Thus, one of the first steps in epidermal cell differentiation is fate specification, whereby each cell adopts either the root-hair or the hairless cell fate. In Arabidopsis as in other members of the Brassicacea, cell fate specification in the root epidermis is associated with the cell's position. Root-hair cells are always located over the junction between adjacent cortical cells (Bunning 1951; Dolan et al. 1993). It is likely that immature epidermal cells receive and respond to positional information that influences their fate. Recently, Arabidopsis mutants have been identified with an abnormal distri- bution of root-hair and hairless epidermal cells. These may define genes whose products are involved in cell fate specification (M. Galway, J. Masucci and J. Schiefelbein, unpublished results).

Several mutants of Arabidopsis alter the morphogenesis of the root-hair cells. The first visible sign of root-hair formation is the swelling of the epidermal cell at the apical end (the site of hair emergence). Two mutants have been identified that produce an abnormally large swelling. These mutants, rhdl (Schiefelbein \& Somerville 1990) and rebl (Baskin et al. 1992), may be defective in the mechanism of localized cell expansion.

Following hair emergence, root-hair cell morphogenesis involves elongation by cell wall addition at the root-hair tip. This type of polarized cell expansion, known as tip growth, also occurs during pollen tube growth. Several loci required for normal root-hair elongation have been identified in Arabidopsis. Mutations in the RHD2 locus result in very short hairs that fail to elongate, indicating that the RHD2 gene is required for the initiation of tip growth (Schiefelbein \& Somerville 1990). Interestingly, the lack of root hairs in rhd 2 mutants does not have a noticeable impact on whole plant growth, suggesting that root hairs are not required for growth under the experimental conditions used (Schiefelbein \& Somerville 1990). Mutations in any one of three loci (RHD3, RHD4 and $T I P 1)$ lead to root hairs of an abnormal shape. In each instance, the cell phenotype indicates a defect in the regulation of the orientation or extent of cellular expansion during tip growth (Schiefelbein \& Somerville 1990; Schiefelbein, Shipley \& Rowse 1992). The tipl mutant is particularly interesting because it is the only root-hair mutant that also has a defect in pollen-tube growth (Schiefelbein et al. 1992).

\section{MUTATIONS THAT AFFECT ROOT RESPONSES TO ENVIRONMENTAL STIMULI}

The direction and extent of root growth are largely determined by environmental factors. Like other plant organs, roots are able to sense and respond to a variety of environmental stimuli. These stimuli include gravity (gravitropic response), light (phototropic response), physical obstacles (thigmotropic response), water (hydrotropic response), temperature (thermotropic response) and certain ions (chemotropic response). There are many unanswered questions related to these responses: How are the stimuli perceived? What plant signalling/messenger systems are involved? How do the roots alter their growth pattern? For some of these responses, the isolation of mutants in Arabidopsis has been useful in identifying genes required for the perception of, or response to, environmental stimuli.

Several mutants of Arabidopsis alter root gravitropism. Normally, Arabidopsis roots direct their growth toward a gravity vector (positive gravitropism) (Fig. 3). Mutations in any one of at least five loci cause plant roots to respond abnormally to gravity or not respond at all. These five loci are auxl (Mirza et al. 1984), dwf (Mirza et al. 1984), agrl 
(a)

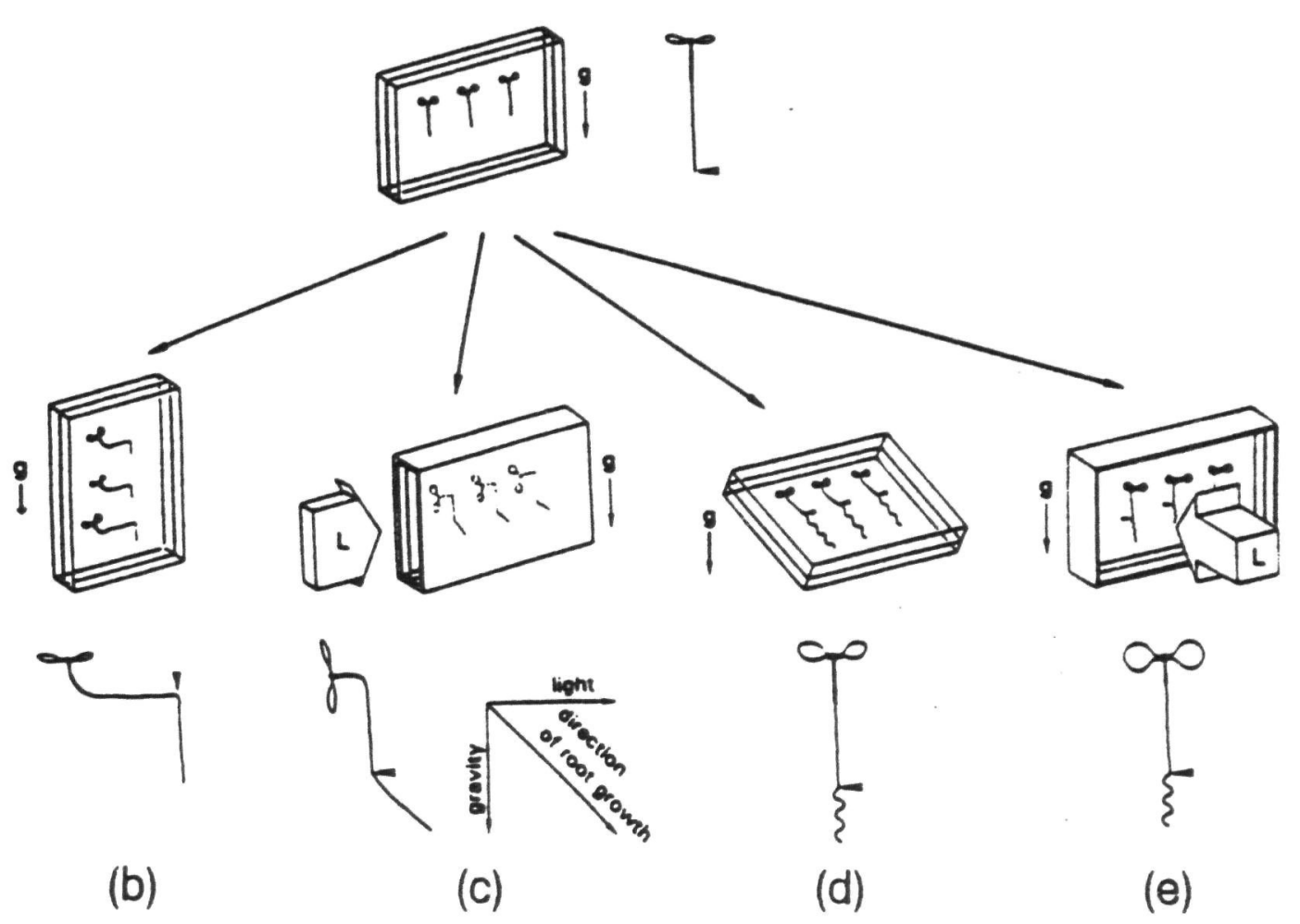

Figure 3. Genetic screens for tropism mutants: (a) seedlings growing on a vertically oriented petri plate; (b) plate is rotated $90^{\circ}$ and roots respond to gravity vector by changing direction of growth, mutants do not respond and continue to grow in the same direction; (c) seedlings are illuminated from one side, wild-type roots respond by growing away from light source but continue to also respond to gravity vector, vutants do not respond to one or both stimuli; (d) hard agar plates are inclined at $45^{\circ}$, wild-type roots attempt to avoid the obstacle of the hard agar by rotating away from the surface then respond to the gravity vector by rotating toward the surface, this results in a wavy pattern of root growth, mutants lack or have abnormal wave patterns; (e) wavy root growth also occurs when the plate is vertical and illuminated from the front by blue light. [From Okada \& Shimura (1992), Cell 70, 369-372. Copyright 1992, Cell Press.]

(Bell \& Maher 1990), axrl (Estelle \& Somerville 1987), and $\operatorname{axr} 2$ (Wilson et al. 1990). These studies implicate the plant hormone auxin in the gravitropic response, since four of the mutants ( $d w f$, auxl, axrl and axr2) exhibit an auxinresistant phenotype.

The ability of growing roots to avoid physical obstacles in their path has also been analysed through the isolation of Arabidopsis mutants. Thigmotropism mutants were identified by growing mutagenized populations of seedlings on agar plates tilted at $45^{\circ}$ to the vertical. In the wild-type, the following sequence of events takes place under these conditions: (1) roots initially attempt to grow downward; (2) they encounter the 'obstacle' (the hard agar surface); (3) they try to avoid the 'obstacle' by rotating their direction of growth; (4) the roots eventually attempt to grow downward again (due to the gravitropic response) and the cycle is repeated (Okada \& Shimura 1990). This periodic obstacle-avoidance response (growth away from and toward the agar) results in a 'wavy' root phenotype (Fig. 3). Mutant seedlings were identified because they exhibited an altered root growth pattern, such as roots with no waves (wavl and wav6), roots with waves of an abnormal shape (wav2, wav3 and wav4), or roots that continuously spiral in a clockwise orientation (wav5) (Okada \& Shimura 1990). Two of these mutants also exhibited abnormal root gravitropism, and complementation tests showed that wav5 is allelic to auxl and wav6 is allelic to agrl.

Root phototropism has been examined by isolating mutants that respond abnormally to light (Fig. 3). In wild- type Arabidopsis, the roots grow away from a light source. Roots of the rptl and rpt 2 mutants continue to grow downward (no response) after receiving illumination from one side (Okada \& Shimura 1992a; Okada \& Shimura 1992b). Interestingly, the shoots of $r p t l$ and rpt2 mutants exhibit normal shoot phototropism, which indicates that the RPTI and $R P T 2$ genes are specifically required for phototropism in roots.

\section{FUTURE DIRECTIONS IN THE APPLICATION OF GENETICS TO THE UNDERSTANDING OF PLANT DEVELOPMENT AND PHYSIOLOGY}

The simple structure and developmental profile of the Arabidopsis root have proven to be major aids in the interpretation of mutant phenotypes. Mutations that affect most stages of development have been isolated and have already provided insight into embryonic root formation, root cell expansion and root hair differentiation. Innovative screens have been used to identify mutations that affect the response of the root to certain environmental stimuli.

In the near future, we can expect to see the isolation of many more mutations that affect root development. Mutations that disrupt the differentiation of tissues other than the epidermis have not yet been reported. To identify all genes involved in root formation, new strategies such as enhancer trapping will have to be employed that can detect genes that are functionally redundant or whose mutation results in early lethality. 
Roots exhibit a remarkable range of responses to environmental stimuli. There are numerous possibilities for genetic analysis in this area. Imaginative screens for mutations in the response to gradients of inorganic compounds, to water gradients or to microbial pathogens could identify important genes.

The molecular characterization of root development genes should lead to a new wave of information. Sequence homologies frequently indicate probable functions. However, there will be some aspects of root development such as cell expansion that have not been well characterized in any organism. The genes that regulate these processes may have novel sequences. Indeed, one of the great attractions of the root is the feasibility of exploring fundamental problems of development and physiology that have proven intractable in other systems.

\section{ACKNOWLEDGMENTS}

We would like to thank Moira Galway, James Masucci and Daphne Foreman for helpful suggestions on the manuscript. The work in the laboratory of J.W.S. was supported by a grant (PCM-9004568) from the NSF. The work in the laboratory of P.N.B. was supported by a grant (GM43778) from the NIH.

\section{REFERENCES}

Barlow P.W. (1976) Towards an understanding of the behavior of root meristems. Journal of Theoretical Biology 57, 433-451.

Baskin T.I., Betzner A.S., Hoggart R., Cork A. \& Williamson R.E. (1992) Root Morphology Mutants in Arabidopsis thaliana. Australian Journal of Plant Physiology 19, 427-438.

Bell J.C. \& Maher E.P. (1990) Mutants of Arabidopsis thaliana with abnormal gravitropic responses. Molecular and General Genetics 220, 289-293.

Benfey P.N., Linstead P.J., Roberts K., Schiefelbein J.W., Hauser M.-T. \& Aeschbacher R.A. (1993) Root development in Arabidopsis: four mutants with dramatically altered root morphogenesis. Development 119, 57-70.

Berleth T. \& Jurgens G. (1993) The role of the monopteros gene in organising the basal body region of the Arabidopsis embryo. Development 118, 575--587.

Bunning E. (1951) Ober die differenzierungsvorgange in der cruciferenwurzel. Planta 39, 126-153.

Clowes, F.A.L. (1958) Development of quiescent centres in root meristems. New Phytologist 57, 85-88.

Dolan L., Janmaat K., Willemsen V., Linstead P., Poethig S., Roberts K. \& Scheres B. (1993) Cellular Organization of the Arabidopsis thaliana Root. Development 119, 71-84.

Estelle M.A. \& Somerville C. (1987) Auxin-resistant mutants of Arabidopsis thaliana with an altered morphology. Molecular and General Genetics 206, 200-206.
Feldman L.J. (1984) The Development and Dynamics of the Root Apical Meristem. American Journal of Botany 71, 1308-1314.

Giddings T.H. \& Staehelin L.A. (1988) Spatial relationship between microtubules and plasma-membrane rosettes during the deposition of primary wall microfibrils in Closterium sp. Planta 173, 22-30.

Gunning B.E.S. (1982) The root of the water fern Azolla: cellular basis of development and multiple roles for cortical microtubules. In Developmental Order: Its Origin and Regulation (eds B. Subtelmy and P. B. Green), pp. 379-421. Alan R. Liss Inc., New York, NY.

Hauser M.-T. \& Benfey P.N. (1993) Genetic Regulation of Root Expansion in Arabidopsis thaliana. In VII ${ }^{\text {th }}$ NATO-ASI on Plant Molecular Biology. 'Molecular-Genetic analysis of Plant Development and Metabolism' (eds P. Pugdomenech \& G. Coruzzi), in press. Springer-Verlag, Berlin.

Jurgens G., Mayer U., Torres Ruiz R.A., Berleth T. \& Misera S. (1991) Genetic analysis of pattern formation in the Arabidopsis embryo. Development Supplement 1, 27-38.

Mayer U., Torres Ruiz R.A., Berleth T., Misera S. \& Jurgens G. (1991) Mutations affecting body organization in the Arabidopsis embryo. Nature 353, 402-407.

Mayer U., Buttner G. \& Jurgens G. (1993) Apical-basal pattern formation in the Arabidopsis embryo: studies on the role of the gnom gene. Development 117, 149-162.

Mirza J.I., Olsen G.M., Iversen T.-H. \& Maher E.P. (1984) The growth and gravitropic responses of wild-type and auxin-resistant mutants of Arabidopsis thaliana. Physiological Plantrum 60, 516-522.

Okada K. \& Shimura Y. (1990) Reversible root tip rotation in Arabidopsis seedlings induced by obstacle-touching stimulus. Science 250, 274-276.

Okada K. \& Shimura Y. (1992a) Mutational analysis of root gravitropism and phototropism of Arabidopsis thaliana seedlings. Australian Journal of Plant Physiology 19, 439-448.

Okada K. \& Shimura Y. (1992b) Aspects of recent developments in mutational studies of plant signaling pathways. Cell 70, 369372.

Scheres B., Willemsen V., Janmaat K., Wolkenfelt H., Dolan L. \& Weisbeek P. (1993) Analysis of Root Development in Arabidopsis thaliana. In VII $^{\text {th }}$ NATO-ASI on Plant Molecular Biology. 'Molecular-Genetic analysis of Plant Development and Metabolism' (eds P. Pugdomenech \& G. Coruzzi), in press. Springer-Verlag, Berlin.

Schiefelbein J.W., Shipley A. \& Rowse P. (1992) Calcium influx at the tip of growing root hair cells of Arabidopsis thaliana. Planta 187, 455-459.

Schiefelbein J.W. \& Somerville C. (1990) Genetic Control of root hair development in Arabidopsis thaliana. Plant Cell 2, 235243.

Wilson A.K., Pickett F.B., Turner J.C. \& Estelle M.A. (1990) A dominant mutation in Arabidopsis confers resistance to auxin, ethylene and abscisic acid. Molecular and General Genetics 222, 377-383.

Received 8 November 1993 
This document is a scanned copy of a printed document. No warranty is given about the accuracy of the copy. Users should refer to the original published version of the material. 